\title{
OPTIMIZACIÓN DEL PROCESO DE EMPAQUE DE LA LÍNEA DE PASTILLAS
}

\author{
Diomar Aurora Dorado, Julián David Cifuentes, Alexander Lozano y Carlos Andrés Nieto \\ Institución Universitaria Antonio José Camacho \\ Recibido: 20/05/2020. Aprobado: 05/10/2020 \\ Cómo citar este artículo: \\ Dorado, D.A., Cifuentes, J.D., Lozano, A. y Nieto, C.A. (2020). Optimización del proceso de empaque de la línea de \\ pastillas. Revista Sapientía, 12 (24), 46-61.
}

\section{RESUMEN}

Mejorar constantemente los procesos ha sido siempre una necesidad permanente en las empresas para satisfacer a los clientes y lograr ser más competitivas. Para obtener estos objetivos las organizaciones buscan diferentes alternativas que no siempre brindan los resultados esperados. Por lo tanto, las soluciones deben ser mucho más eficientes llevando a aprovechar al máximo los recursos económicos, mano de obra y el tiempo, encaminado a mejorar la satisfacción de los clientes con los productos y servicios. En este proyecto el objetivo es proponer varias soluciones que incrementen la eficiencia en el proceso de empaque en la línea de Pastillas x 2, donde se ha evidenciado que los tiempos de empaque del producto mencionado están generando la pérdida de hora hombre, la cual podría ser utilizada en otro proceso dentro de la misma área. Para el desarrollo del proyecto se realizará un análisis de restricciones para encontrar el cuello de botella que presenta actualmente el proceso de empaque en la línea de Pastillas. Adicionalmente, con el análisis se procede a tomar los métodos y tiempos, incluyendo el análisis de fatiga, para determinar así las fallas que se tienen en el proceso actual.

A partir de los análisis realizados se plantean los objetivos propuestos para la optimización del proceso de empaque de la línea de Pastillas tabletas x 2, involucrando a los operarios mediante una capacitación que les dé a conocer los objetivos propuestos en este proyecto.

\section{PALABRAS CLAVE}

Cuello de botella, métodos y tiempos, optimización.

\section{ABSTRACT}

Constantly improving processes has always been a permanent need in companies to satisfy customers and become more competitive. To obtain these objectives, organizations look for different alternatives that do not always provide the expected results. Therefore, solutions must be much more efficient, making the most of economic resources, labor and time, aimed at improving customer satisfaction with products and services. In this project, the objective is to propose several solutions that increase the efficiency in the packaging process in the Pastillas $x 2$ line, where it has been shown that the packaging times of the mentioned product are generating the loss of man-hour, which could be used in another process within the same area. For the development of the project, a restriction analysis 
will be carried out to find the bottleneck that currently presents the packaging process in the Pastillas line. Additionally, the analysis proceeds to take the methods and times, including the fatigue analysis, to determine the failures that exist in the current process.

Based on the analyzes carried out, the proposed objectives for the optimization of the packaging process of the Pastillas tablets $\mathrm{x} 2$ line are proposed, involving the operators through training that makes them aware of the objectives proposed in this project.

\section{KEYWORDS}

Bottleneck, methods and times, optimization.

\section{INTRODUCCIÓN}

El desarrollo del presente proyecto se dio como respuesta a la iniciativa del supervisor de empaque de la empresa farmacéutica. El área de empaque carece de un proceso eficiente, debido a que la máquina WRAP-ADE I (la cual realiza el corte de las tabletas de pastillas por 2 unidades) realiza 108 cortes por minuto y la capacidad de la línea de empaque no cumple con los tiempos determinados para el mismo; en esta empresa no se desarrollan las actividades de forma organizada para cumplir eficientemente con el proceso de empaque, para empacar un lote de 500.000 tabletas se demoran actualmente 40 horas, significa que son 6,5 turnos de trabajo, para este se utilizan 18 operarios distribuidos de la siguiente manera: 1 operario organizador, 11 operarios en la línea de empaque en plegadiza, 2 operarios que pesan el producto, 2 empaque en corrugada y 1 auxiliar de línea que organiza las corrugadas en la estiba.

El presente estudio se justifica desde el punto de vista práctico porque propone al problema planteado una solución, cuyos pasos se describen en los objetivos de este documento.
El objetivo de la investigación es optimizar y restructurar el proceso de empaque de la línea de pastillas tabletas con presentación de caja por 2 tabletas en la empresa farmacéutica con el fin de determinar las causas que ocasionan los cuellos de botella y las pérdidas de tiempo en el proceso de empaque.

\section{MARCO TEÓRICO}

\section{Estudio de métodos}

El estudio de trabajo es el examen sistemático de los métodos preferentes para llevar a cabo una operación, con el fin de mejorar una optimización de recursos y desarrollar normas de rendimiento con respeto a las actividades que forman parte del proceso productivo.

\section{Propósito en el estudio del método del trabajo}

- Evaluar el comportamiento del trabajador.

- Planear la necesidad de la fuerza de trabajo.

- Determinar la capacidad disponible.

\section{VSM}

El Value Stream Mapping es una herramienta clave dentro de la metodología Lean Manufacturing y es un diagrama que se utiliza para visualizar, analizar y mejorar el flujo de los productos y de la información dentro de un proceso de producción, desde el inicio del proceso hasta la entrega al cliente.

\section{Metodología de 5'S}

La metodología de las $5 S$ se creó en Toyota en los años 60 y agrupa una serie de actividades que se desarrollan con el objetivo de crear condiciones de trabajo que permitan la ejecución de labores de forma organizada, ordenada y limpia. Dichas condiciones se crean a través de reforzar los buenos hábitos de comportamiento e interacción social, creando un entorno de trabajo eficiente y productivo.

\section{Lead time}

Los plazos de entrega se ven afectados por muchos factores, incluidos la capacidad, la carga, el 
procesamiento por lotes y la programación, y ellos mismos afectan muchos aspectos de los costos y el control. Además, también se analizan los modelos que incorporan plazos de entrega en la planificación de la capacidad y las decisiones de diseño y se discuten algunas direcciones para la investigación.

\section{Análisis de fatiga}

También llamado análisis ergonomía, se encarga de que todas las condiciones de trabajo estén óptimas para que el operario pueda realizar la labor. Para este manejan unas tablas de coeficiente de descanso que queda determinado por los factores ergonómicos, es decir, entorno y herramientas o máquinas.

\section{DESARROLLO DE LA METODOLOGÍA}

\section{Estudio de métodos y tiempos}

Para realizar un análisis más profundo y determinar en realidad que lo anteriormente mencionado es el cuello de botella, se procedió a tomar los tiempos en la línea de empaque en tiempo real. Tiempos que es posible observar en la Tabla 1.

Tabla 1. Estudio de tiempos Pastillas por 2 tabletas

\begin{tabular}{|c|c|c|c|c|c|c|c|c|c|c|c|c|c|c|c|c|c|c|c|}
\hline \multicolumn{20}{|c|}{ Estudio de Tiempos } \\
\hline \multirow{6}{*}{$\begin{array}{l}\text { Descripción de la } \\
\text { actividad } \\
\text { Personal } \\
\text { Departamento/área } \\
\text { Producto }\end{array}$} & & & & & & & & & Dia & Mes & Año & & & & & & & & \\
\hline & & & & & & & & & 1 & 4 & 2019 & & & & & & & & \\
\hline & \multicolumn{11}{|c|}{ Empaque Noraver Pastilla Cereza } & & & & & & & & \\
\hline & \multicolumn{19}{|c|}{$\begin{array}{l}\text { La línea se conforma por } 18 \text { personas (1 operario organizador, } 9 \text { operarios empacan en plegadiza, } 2 \text { operarios en pesa, } 2 \text { operarios ponen sello y } 2 \text { empaca en corrugada, } 2 \\
\text { ayudante de Línea) }\end{array}$} \\
\hline & \multicolumn{11}{|c|}{\begin{tabular}{|l|l|} 
Efervecentes \\
\end{tabular}} & & & & & & & & \\
\hline & \multicolumn{11}{|c|}{ Noraver Pastilla Cereza X2 } & & & & & & & & \\
\hline \multicolumn{2}{|c|}{ No. Actividad } & \multicolumn{2}{|c|}{1} & \multicolumn{2}{|c|}{2} & \multicolumn{2}{|c|}{3} & \multicolumn{2}{|l|}{4} & \multicolumn{2}{|c|}{5} & \multicolumn{2}{|c|}{6} & \multicolumn{2}{|l|}{7} & \multicolumn{2}{|c|}{8} & \multicolumn{2}{|l|}{9} \\
\hline \multicolumn{2}{|c|}{ Actividades } & \multicolumn{2}{|c|}{$\begin{array}{l}\text { OPERARIO } \\
\text { ORGANIZADOR }\end{array}$} & \multicolumn{2}{|c|}{$\begin{array}{l}\text { VERIFICACION } \\
\text { DE LAMINADO }\end{array}$} & \multicolumn{2}{|c|}{$\begin{array}{l}\text { ARMADO DE } \\
\text { PLEGADIZA }\end{array}$} & $\begin{array}{l}\text { INSERTAR TAB } \\
\text { Y CERRAR PLEC }\end{array}$ & $\begin{array}{l}\text { BLETAS } \\
\text { GADIZA }\end{array}$ & $\begin{array}{l}\text { PEC } \\
\text { SELLC } \\
\text { SEGUF }\end{array}$ & $\begin{array}{l}\text { GAR } \\
\text { OS DE } \\
\text { IRIDAD }\end{array}$ & $\begin{array}{c}\text { VERIFIC } \\
\text { DE P }\end{array}$ & $\begin{array}{l}\text { CACION } \\
\text { PESO }\end{array}$ & $\begin{array}{l}\text { ORGANIZACIÓ } \\
\text { CAJA POR } 5 \text { UNII }\end{array}$ & $\begin{array}{l}\text { SN DE } \\
\text { IDADES }\end{array}$ & $\begin{array}{l}\text { EMPAC } \\
\text { CORRU }\end{array}$ & $\begin{array}{l}\text { CAR EN } \\
\text { UGADO }\end{array}$ & ESTIBAR PR & RODUCTO \\
\hline & & acum & $\mathrm{E}$ & acum & $\mathrm{E}$ & acum & $\mathrm{E}$ & acum & $\mathrm{E}$ & $\mathrm{R}$ & E & $\mathrm{R}$ & $\mathrm{E}$ & $\mathrm{R}$ & $\mathrm{E}$ & $\mathrm{R}$ & $\mathrm{E}$ & $\mathrm{R}$ & E \\
\hline & 1 & 2.0 & 2.0 & 3.5 & 3.5 & 15.0 & 15.0 & 2.5 & 2.5 & 1.9 & 1.9 & 3.7 & 3.7 & 5.6 & 5.6 & 4.0 & 4.0 & 65.0 & 65.0 \\
\hline & 2 & 3.8 & 1.8 & 6.9 & 3.4 & 33.4 & $\begin{array}{l}18.4 \\
\end{array}$ & 5.4 & 2.9 & 3.6 & 1.7 & 8.0 & 4.4 & 11.6 & 6.0 & 7.4 & 3.4 & 135.9 & 70.9 \\
\hline & 3 & 5.8 & 2.0 & 8.9 & 2.0 & 44.3 & 10.9 & 7.5 & 2.1 & 4.8 & 1.2 & 12.1 & 4.1 & 16.9 & 5.3 & 11.0 & 3.6 & 220.8 & 84.8 \\
\hline & 4 & 8.1 & 2.3 & 12.5 & 3.5 & 61.7 & 17.4 & 10.4 & 2.9 & 6.0 & 1.1 & \begin{tabular}{|l|l|}
14.7 \\
\end{tabular} & 2.6 & 21.8 & 4.9 & \begin{tabular}{|l|}
14.1 \\
\end{tabular} & 3.1 & 303.2 & 82.5 \\
\hline & 5 & 10.1 & 2.0 & \begin{tabular}{|l|}
14.7 \\
\end{tabular} & 2.2 & 75.9 & 14.2 & 12.8 & 2.4 & 7.9 & 1.9 & 17.8 & 3.1 & 27.4 & 5.6 & 17.1 & 3.0 & 387.3 & 84.1 \\
\hline & 6 & 12.0 & 1.9 & \begin{tabular}{|l|}
17.1 \\
\end{tabular} & 2.4 & 89.3 & 13.4 & 15.2 & 2.4 & 9.1 & 1.2 & 22.6 & 4.8 & 32.9 & 5.5 & 20.2 & 3.1 & 458.2 & 70.9 \\
\hline & 7 & 14.0 & 2.0 & 19.3 & 2.2 & 104.9 & 15.6 & $\begin{array}{l}17.7 \\
\end{array}$ & 2.4 & 10.8 & 1.7 & 25.2 & 2.6 & 38.0 & 5.1 & 23.8 & 3.6 & 519.9 & 61.7 \\
\hline$\frac{0}{0}$ & 8 & 16.0 & 2.0 & 22.4 & 3.1 & 118.6 & $\begin{array}{l}13.7 \\
\end{array}$ & 20.0 & 2.3 & 12.0 & 1.3 & \begin{tabular}{|l|}
28.3 \\
\end{tabular} & 3.1 & 43.4 & 5.5 & 27.6 & 3.8 & 594.2 & 74.4 \\
\hline & 9 & 17.8 & 1.9 & 26.1 & 3.7 & 129.4 & 10.8 & 22.1 & 2.1 & 13.2 & 1.2 & \begin{tabular}{|l|}
32.3 \\
\end{tabular} & 4.0 & 49.3 & 5.9 & 30.8 & 3.1 & 670.7 & 76.5 \\
\hline & 10 & $\begin{array}{l}19.7 \\
\end{array}$ & 1.9 & 30.0 & 3.9 & 141.9 & 12.6 & 24.4 & 2.3 & 15.0 & 1.8 & 36.6 & 4.3 & 54.6 & 5.3 & 34.0 & 3.3 & 735.0 & 64.2 \\
\hline & 11 & 21.7 & 2.0 & 33.0 & 3.0 & 161.4 & 19.5 & 26.8 & 2.4 & 17.0 & 2.0 & 41.3 & 4.7 & 60.0 & 5.4 & 37.3 & 3.3 & 817.7 & 82.7 \\
\hline & 12 & 24.2 & 2.5 & 36.7 & 3.7 & 179.2 & 17.8 & 29.7 & 2.9 & 18.9 & 1.9 & 43.3 & 2.0 & 64.2 & 4.3 & 41.2 & 3.9 & 896.4 & 78.7 \\
\hline & 13 & 26.2 & 2.0 & 39.7 & 3.1 & 198.1 & 18.9 & 31.9 & 2.2 & 20.2 & 1.3 & 46.3 & 3.0 & 69.4 & 5.2 & 45.1 & 3.9 & 973.9 & 77.5 \\
\hline & 14 & 28.2 & 2.0 & 41.7 & 2.0 & 211.8 & 13.7 & 34.1 & 2.2 & 21.6 & 1.4 & \begin{tabular}{|l|}
48.8 \\
\end{tabular} & 2.6 & 73.5 & 4.1 & 48.6 & 3.5 & 1041.5 & 67.5 \\
\hline & 15 & 30.2 & 2.0 & 45.6 & 3.9 & 222.3 & 10.5 & 36.5 & 2.4 & 22.6 & 1.1 & 53.1 & 4.3 & 79.0 & 5.5 & 52.4 & 3.8 & \begin{tabular}{l|l}
8 & 1107.0 \\
\end{tabular} & 65.5 \\
\hline OBSERVACIONES & Tiempo Total & 30.15 & 30.2 & 45.63 & 45.63 & 222.31 & 222.31 & 36.45 & 36.45 & 22.64 & 22.64 & 53.13 & 53.13 & 78.99 & 78.99 & 52.42 & 52.42 & 1106.99 & 1106.99 \\
\hline & No. Ciclos & & 15 & & 15 & & 15 & & 15 & & 15 & & 15 & & 15 & & 15 & & 15 \\
\hline & Unid/ciclo & & 1 & & 2 & & 1 & & 1 & & 1 & & 1 & & 1 & & 1 & & 8 \\
\hline & $\begin{array}{l}\text { Velocidad } \\
\text { (unid/min) }\end{array}$ & & 29.85 & & 39.45 & & 4.05 & & 24.69 & & 39.75 & & 16.94 & & 11.39 & & 17.17 & & 6.50 \\
\hline & Tiempo promedio & & 2.01 & & 3.04 & & 14.82 & & 2.43 & & \begin{tabular}{|l|}
1.51 \\
\end{tabular} & & 3.54 & & 5.27 & & 3.49 & & 73.80 \\
\hline & Coeficiente variac & & $9 \%$ & & $23 \%$ & & $20 \%$ & & $11 \%$ & & $22 \%$ & & $25 \%$ & & $10 \%$ & & $10 \%$ & & $11 \%$ \\
\hline & \% valoración & & $10 \%$ & & $13 \%$ & & $18 \%$ & & $17 \%$ & & $21 \%$ & & $21 \%$ & & $10 \%$ & & $11 \%$ & & $10 \%$ \\
\hline & Tiempo normal & & 2.21 & & 3.44 & & 17.49 & & 2.84 & & 1.83 & & 4.29 & & 5.79 & & 3.88 & & 81.18 \\
\hline & $\%$ suplementos & & $20 \%$ & & $20 \%$ & & $20 \%$ & & $17 \%$ & & $16 \%$ & & $17 \%$ & & $14 \%$ & & $14 \%$ & & $16 \%$ \\
\hline & Tiempo estandar & & 2.65 & & 4.12 & & 20.99 & & 3.33 & & 2.12 & & 5.01 & & 6.60 & & 4.42 & & 94.17 \\
\hline & Desvicion estandar & & 0.2 & & 0.7 & & 3.0 & & 0.3 & & 0.3 & & 0.9 & & 0.5 & & 0.3 & & 7.9 \\
\hline & Tiempo maximo & & 2.50 & & 19.51 & & 3.91 & & 2.94 & & 1.96 & & 4.77 & & 5.95 & & 4.00 & & 84.83 \\
\hline & Tiempo minimo & & 1.80 & & 10.51 & & 2.01 & & 2.11 & & 1.05 & & 2.01 & & 4.06 & & 3.03 & & 61.67 \\
\hline
\end{tabular}


En la tabla anterior podemos evidenciar los tiempos más representativos que están generando problemas en la línea de empaque de Pastillas. A continuación, en la Tabla 2, se detalla el coeficiente de variación de los 4 tiempos más importantes:

Tabla 2. Coeficiente de variación de los 4 tiempos más importantes.

\begin{tabular}{lll}
\hline Actividades & Tiempo $(\mathbf{s})$ & Coeficiente de Variación \\
\hline Armado de plegadiza & 20,99 & $20 \%$ \\
\hline Pegar sellos de seguridad & 2,12 & $22 \%$ \\
\hline Verificación de peso & 5,01 & $25 \%$ \\
\hline Verificación de laminado & 4,00 & $23 \%$ \\
\hline Insertar Tabletas en Plegadiza y cerrar plegadiza & 3,07 & $11 \%$ \\
\hline
\end{tabular}

Con los datos anteriores representados en la Tabla 2 se logra cuantificar la variación o dispersión de los datos. Así como identificar cuáles son las causas más propensas a que los datos suministrados por el coeficiente de variación generen una dispersión de los tiempos calculados, con estas posibles fallas en la en la operación es posible hacer un análisis más adecuado para una estandarización del proceso.

En la Tabla 3 se identifica la cantidad de tabletas que se deberían empacar en un turno de 8 horas, según la capacidad teórica de la máquina envasadora.

Tabla 3. Cantidad de empaque según la capacidad teórica de la máquina

\begin{tabular}{|c|c|c|c|}
\hline \multicolumn{4}{|c|}{ Máquina Encelofanadora WRAP-ADE I } \\
\hline \multicolumn{2}{|l|}{ Velocidad Máquina } & \multicolumn{2}{|l|}{216 Tabletas x Min. } \\
\hline Producto & Tiempo & Cantidad & Porcentaje \\
\hline \multirow[t]{8}{*}{ Pastillas x2 } & 1 Hora & 12960 & $13 \%$ \\
\hline & 2 Hora & 25920 & $25 \%$ \\
\hline & 3 Hora & 38880 & $38 \%$ \\
\hline & 4 Hora & 51840 & $50 \%$ \\
\hline & 5 Hora & 64800 & $63 \%$ \\
\hline & 6 Hora & 77760 & $75 \%$ \\
\hline & 7 Hora & 90720 & $88 \%$ \\
\hline & 8 Hora & 103680 & $100 \%$ \\
\hline Tabletas por turno & & 103,680 & \\
\hline
\end{tabular}




\section{ANÁLISIS DE TIEMPOS Y MOVIMIENTOS}

Para realizar este análisis primero debemos conocer el proceso de empaque desde su inicio hasta el final, desarrollando el diagrama de flujo del proceso (véase en la Figura 5).

DIAGRAMA DE OPERACIONES LÍNEA PASTILLAS POR 2 TABLETAS

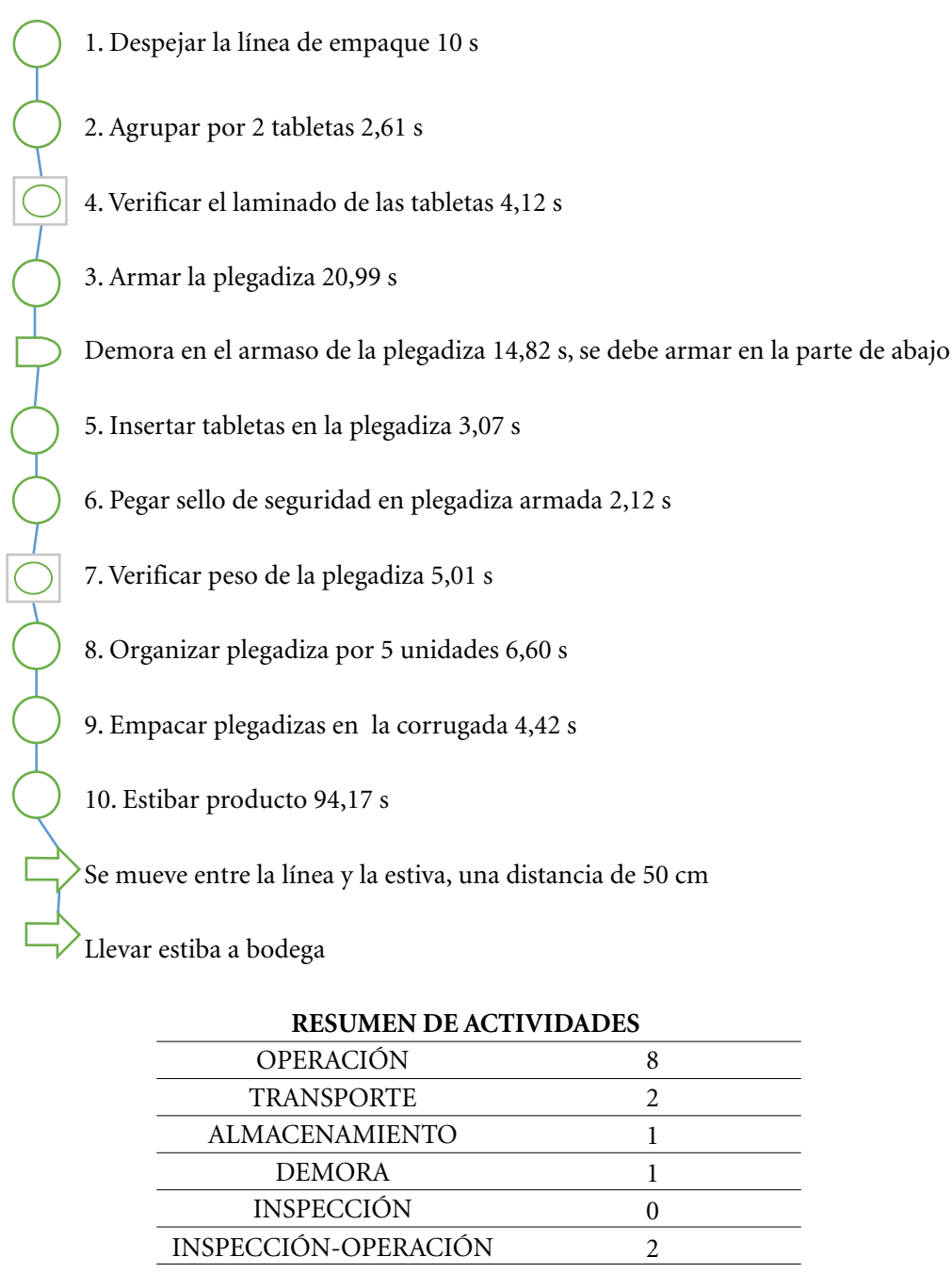

Figura 1. Diagrama de proceso de empaque

\section{PROPUESTA DE MEJORAMIENTO}

Basándonos en el anterior estudio, se realiza la propuesta de optimizar y restructurar el proceso de empaque de la línea de pastillas tabletas con presentación de caja por 2 tabletas en la empresa farmacéutica. Las fases son las siguientes: 
- Fase 1: Identificar los problemas que presenta el proceso de empaque actual.

- Fase 2: Realizar una propuesta para mejorar la eficiencia del proceso de empaque.

- Fase 3: Evaluar el impacto de la propuesta de la optimización del proceso de empaque.

- Fase 4: Realizar la relación de costo vs beneficio del proyecto

\section{FASE 1: IDENTIFICAR LOS PROBLEMAS QUE PRESENTAN EL PROCESO EMPAQUE.}

\section{Identificación de problemas}

Al realizar un análisis por el tiempo promedio de cada actividad (véase en la Tabla 1) se evidencia que las actividades que se encuentran fuera de control son las siguientes:

- Armado de plegadiza con $20 \%$

- Verificación del laminado $23 \%$

- Insertar tabletas en plegadiza $11 \%$

- Pegar sellos de seguridad $22 \%$

- Verificación de peso $25 \%$
Teniendo en cuenta lo observado en el estudio de tiempos generados en la línea de Pastillas por dos tabletas, evaluamos más al detalle los tiempos por ciclo de cada una de las actividades.

\section{Armado de plegadiza}

Esta actividad tiene un coeficiente de variación de $20 \%$, en los tiempos 19,$51 ; 18,86$ y 18,44 . Se encuentran por fuera del límite de especificación superior, debido a que los operarios no cuentan con la experticia suficiente para realizar dicha operación (véase Gráfico 12). En cuanto a los tiempos que se encuentran dispersos del promedio 14,80 , esto obedece a que la plegadiza no es semiautomática (que se puede armar solo girando la plegadiza al lado derecho), por lo que el operario debe realizar la operación de cerrado manual en ambos extremos. Como se evidencia en el gráfico, hay 3 tiempos por debajo del límite inferior, esto se debe a que en el momento de tomar los tiempos había personas con experiencia en el proceso.

Para realizar un análisis más profundo se procedió con un ejercicio de comparación de la plegadiza normal vs la semiautomática, para lo cual se tomaron 15 tiempos de armado de cada plegadiza. En la Figuras 2 se puede ver la diferencia entre cada una. 

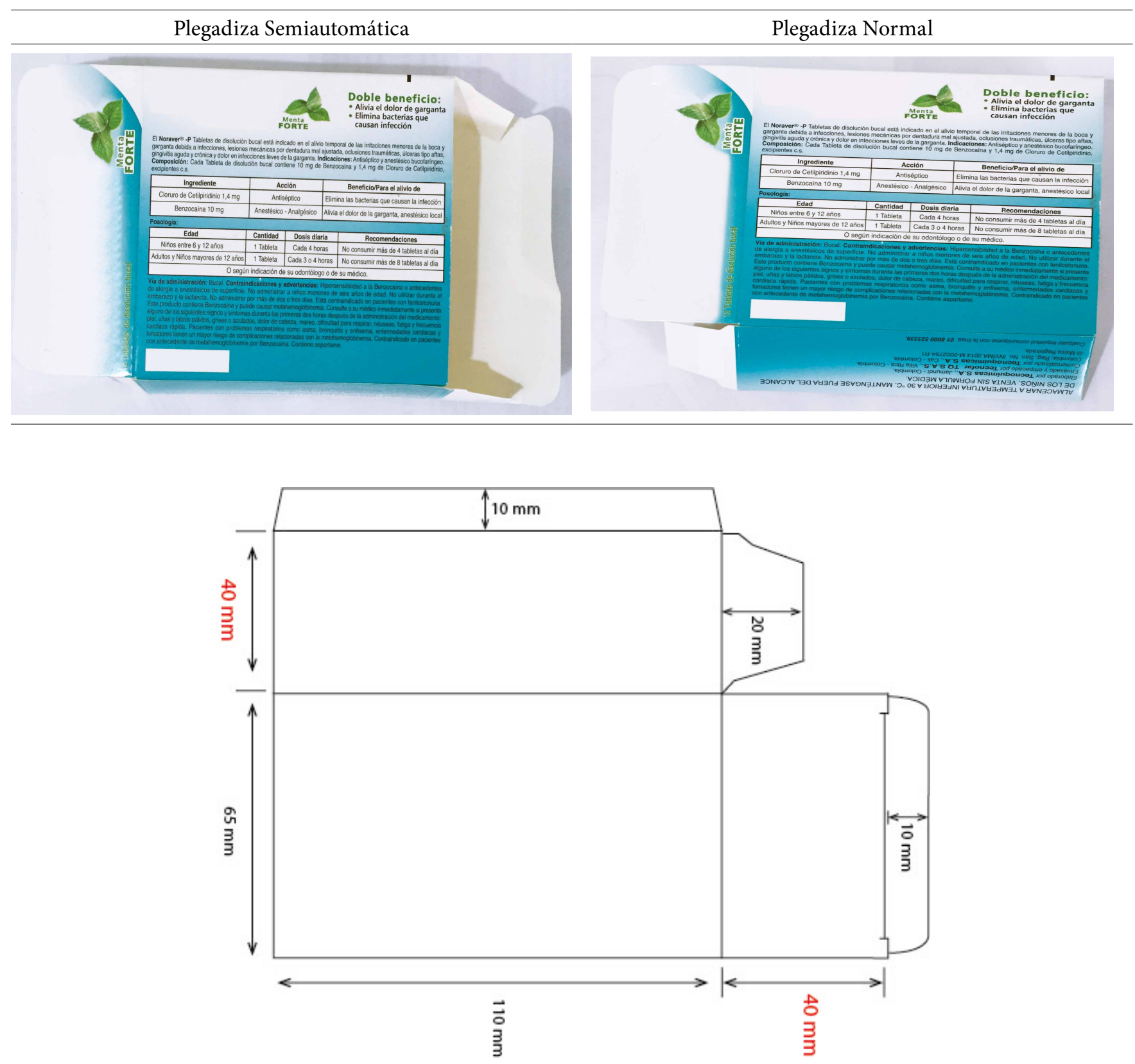

Figura 2. Comparativo de Plegadiza 
Laplegadiza semiautomática tienela partelateralderecha oculta dentro de la caja para solamente dar un giro a la derecha y cerrar ese lado, mientras que la caja normal (la cual utiliza el producto de estudio) debe cerrar el lateral derecho como el izquierdo manualmente.

Tabla 4. Tiempos de plegadiza normal vs semiautomática

\begin{tabular}{ccc}
\hline Ciclos & $\begin{array}{c}\text { Armado plegadiza } \\
\text { insertar y cerrar } \\
\text { plegadiza normal }\end{array}$ & $\begin{array}{c}\text { Armado plegadiza } \\
\text { con cerrado semiau- } \\
\text { tomático }\end{array}$ \\
\hline 1 & 17.5 & 3.5 \\
\hline 2 & 21.3 & 3.8 \\
\hline 3 & 13 & 3.6 \\
\hline 4 & 20.3 & 3.9 \\
\hline 5 & 16.6 & 4 \\
\hline 6 & 15.8 & 3.5 \\
\hline 7 & 18 & 3.6 \\
\hline 8 & 16 & 3.6 \\
\hline 9 & 12.9 & 3.8 \\
\hline 10 & 14.9 & 3.5 \\
\hline 11 & 21.9 & 3.7 \\
\hline 12 & 20.7 & 3.5 \\
\hline 13 & 21.1 & 3.9 \\
\hline 14 & 15.9 & 3.8 \\
\hline 15 & 12.9 & 3.5 \\
\hline Promedio & 17.25 & 3.68 \\
\hline
\end{tabular}

En la Tabla 4 podemos observar que los valores en el armado de la plegadiza normal son altos vs los valores del armado de plegadiza con cierre semiautomático. Esta operación con cierre semiautomático genera un incremento del $21 \%$ en la producción; para hallar este valor se divide el promedio de armado con cierre semiautomático y el promedio de armado de plegadiza y cierre normal.

\section{Verificación del laminado}

Esta actividad tiene el coeficiente de variación en 23\%. Se tienen dos tiempos fuera del límite de especificación 3,85 y 3,91; los otros tiempos se encuentran dispersos entre el promedio, lo cual puede deberse a que los operarios no tienen la destreza necesaria para la verificación: en algunos momentos de la toma de los tiempos había operarios que realizaban movimientos innecesarios como subir las manos con las tabletas.

\section{Insertar tabletas en plegadiza}

Esta actividad tiene un coeficiente de variación del $11 \%$, actualmente se cuenta con 3 ciclos fuera del límite de especificación 2,89 y 2,94 . Si bien su coeficiente no es tan alto, se debe entrar a revisar los ciclos que se pasaron de tiempo.

\section{Recurso humano}

En la situación actual de Farmacéutica SAS se pueden evidenciar y establecer diferentes fallas que retrasan el proceso. De acuerdo con lo evidenciado en la toma de tiempos se establece que:

- El personal no se encuentra alineado con las actividades que deben realizar en la línea, ya que son rotados en diferentes líneas de proceso.

- No existen perfiles definidos por la línea de empaque para los procesos de contratación de personal, que contengan las destrezas y experiencia mínimas requeridas para poder ser operario de empaque $y$, por ende, existe personal laborando que no cuenta con la experiencia suficiente para realizar las labores para las cuales fue contratado.

- Existe mucha rotación de personal en la compañía, lo que hace que el sistema de selección de personal sea cada vez más flexible y que se contrate personal no calificado, lo cual se refleja en los diferentes problemas que se presentan en los procesos del proceso de empaque.

\section{FASE 2: REALIZAR UNA PROPUESTA PARA MEJORAR LA EFICIENCIA DEL PROCESO DE EMPAQUE}

Debido a la situación actual esto nos lleva a pensar que la propuesta para optimizar el proceso productivo se enfoca en dos recursos indispensables que intervienen en el proceso de empaque:

- Recurso Humano

- Recurso Tecnológico 


\section{Recurso humano}

\section{Proceso de contratación}

Teniendo en cuenta que el personal de la compañía es seleccionado por RH y una empresa temporal, es necesario que cada empleado cumpla con la valoración de desempeño realizada en la toma de tiempos (véase las tablas 13 y 14) para la ejecución de las actividades de forma eficiente y eficaz para cumplir con los objetivos planteados en el día a día.

Evaluando este aspecto y teniendo en cuenta los problemas que se presentan actualmente, se propone lo siguiente para el proceso de contratación:

- La creación de un perfil definido para el cargo de Operario de Empaque donde se tenga en cuenta los porcentajes de valoración, que sea incluido dentro del proceso de selección.

- Al ingreso de la persona a la compañía, se debe de enviar a la planta de Jamundí para que se capacite de forma práctica sobre el proceso.

- Por otra parte, proponemos reforzar el programa de plan anual para la capacitación del personal teniendo en cuenta las personas que llevan más años en la compañía. Crear un grupo de mentores que se encarguen del entrenamiento del personal nuevo con el fin de estandarizar los tiempos de empaque de estas personas.

\section{Mejoramiento del método}

Según el análisis realizado en la fase anterior se identificó que la actividad pegado de sello de seguridad tiene un coeficiente de variación del $22 \%$. Para disminuir este porcentaje se realizó un análisis de Benchmarking entre la empresa Farmacéutica y Laboratorios abc, donde la actividad de pegado de sello de seguridad se realiza de la siguiente manera: "toman 3 plegadizas, las giran a $45^{\circ}$ y pegan los 3 sellos de seguridad, les dan la vuelta a las cajas en el mismo ángulo de $45^{\circ}$ grados y pegan los sellos de seguridad del otro lado de la caja”. En Farmacéutica se realiza esta actividad uno a uno, por tal razón se propone cambiar el método actual por el descrito anteriormente y así conseguir un mejor rendimiento en tiempo y costo.

\section{Descripción de la máquina estuchadora DZH-120}

Esta máquina fue cotizada por medio de una empresa China. La DZH-120 es un equipo de encartonado automático inteligente y continuo, con velocidad máxima de hasta 70-120 cajas/min. Esta máquina es adecuada para la alimentación automática y el envasado de alimentos, medicamentos, químicos, diarios, entretenimiento, ferretería y otros artículos. Esta máquina tiene un costo de 24.500 USD.

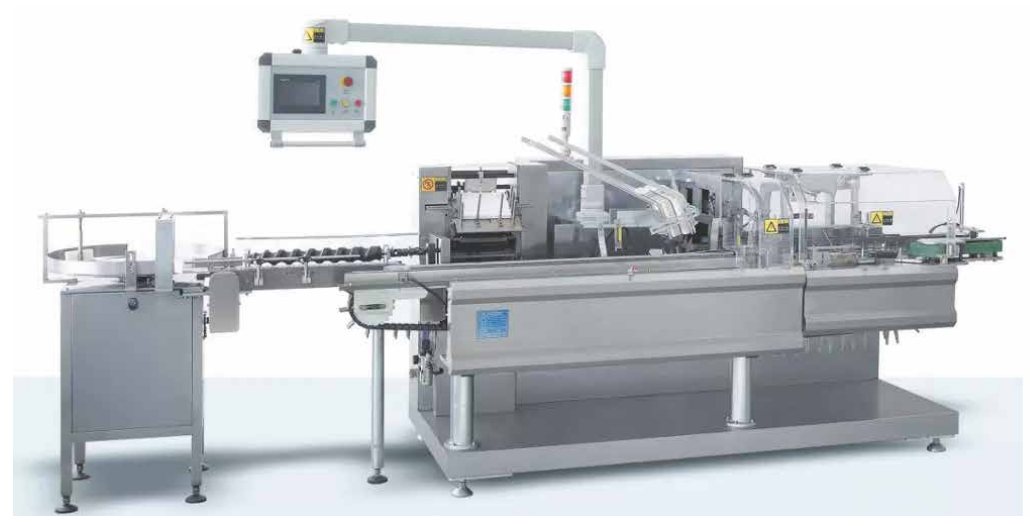

Figura 3. Máquina Estuchadora 
La capacidad de la máquina estuchadora es de 70 a 120 plegadizas/min; se puede configurar en 108 plegadizas/min generando así un balanceo en la línea de empaque. Si se realiza la adquisición de la máquina esta puede empacar un total de 103680 unidades por turno (las cuales salen de la siguiente operación: 108 und /min que entrega la máquina de envase por 480 minutos que equivalen al turno de 8 horas), y la totalidad del lote se empacaría en 4,5 turnos.

\section{Cinturón electrónico dinámico con pesador}

Para minimizar los tiempos de verificación de peso se cotizará un cinturón electrónico dinámico pesador para adecuar a la banda transportadora, el cual tiene un costo de 4.200 USD. Las características del equipo son las siguientes:

\section{ALWㄷㄴㄴ \\ 躍焉}

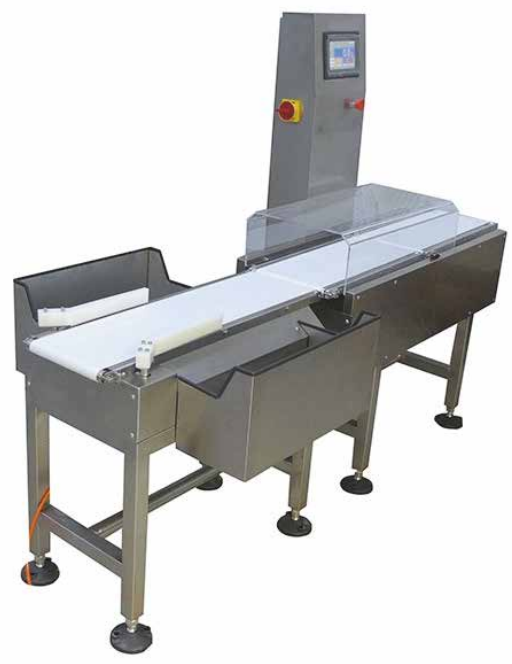

Figura 4. Cinturón Electrónico

Las propuestas de optimización del proceso quedarían de la siguiente manera:

- Adquirir la Máquina Estuchadora con el cinturón dinámico adaptados a la banda transportadora y así automatizar las actividades de la plegadiza y verificación de peso en línea.
- Modificar el diseño de la plegadiza y adquirir solo el cinturón dinámico para adaptar a la banda transportadora.

- Para este último se entrega la propuesta de cambio del diseño de la plegadiza normal por una de cierre semiautomático para generar un incremento en la producción del $21 \%$ (véase en la Tabla 18), debido a que los tiempos de esta operación se ven disminuidos por la facilidad de empaque.

Unidades producidas 81000 por el $21 \%$ de incremento $=98010$ unidades empacadas

\section{FASE 3: EVALUAR EL IMPACTO DE LA PROPUESTA DE LA OPTIMIZACIÓN DEL PROCESO DE EMPAQUE}

Para evaluar el impacto de las propuestas realizadas se tuvo en cuenta el recurso humano y tecnológico con el fin de identificar las mejoras del proceso. Para esta fase se tiene en cuenta las actividades que tienen el coeficiente de variación más alto sobre los cuales se generan las propuestas de valor.

\section{Recurso humano Proceso de contratación}

Con la creación de perfiles para los cargos de Operario de Empaque se puede estandarizar la selección del personal y garantizar que la persona que ingresa cumple con la experiencia necesaria para el cargo.

\section{Mejoramiento del método}

Para la actividad Pegar Sello de Seguridad se realizó un muestreo de 50 unidades con los operarios encargados, donde se pegaron los sellos de la forma actual vs la propuesta, esto permitió determinar lo siguiente:

- Con el método actual se pega 2 sellos de seguridad (1 caja por ambos lados) en 1,51s según el promedio por sello que sería el total de 3,02s la caja completa. 
- Con el método propuesto para pegar los 6 sellos de seguridad (3 cajas por ambos lados) en 1,19s según el promedio por sello, que sería un total de $7,15 \mathrm{~s}$,

Tabla 5. Mejoramiento del método

\begin{tabular}{ll}
\hline \multicolumn{1}{c}{ Método Actual } & \multicolumn{1}{c}{ Método Mejorado } \\
\hline $\begin{array}{l}\text { Pegar Sellos de seguridad en } \\
1 \text { caja }(3,02 \mathrm{~s})\end{array}$ & $\begin{array}{l}\text { Pegar Sellos de Seguridad } \\
\text { en } 3 \text { cajas }(7,15 \mathrm{~s})\end{array}$ \\
\hline
\end{tabular}

En la Tabla 5 se evidencia la diferencia entre los dos métodos, por lo que es posible afirmar que con el método mejorado incrementamos la productividad de la actividad en un $78 \%$.

\section{Recurso tecnológico}

En cuanto al recurso tecnológico el impacto esperado de las propuestas de maquinaria es disminuir los tiempos de operación del proceso, disminuir el personal de la línea y reubicar las personas que sobran de la línea.

\section{Propuesta 1}

Con la compra de la máquina estuchadora DZH120 y la balanza dinámica se disminuirían 11 personas de la línea, las cuales se reubican en el área de efervescentes, ya que el proceso quedaría en su mayoría automatizado. Esta máquina tiene la capacidad para alinearla a la máquina encelofanadora WRAP-ADE I y empacar en línea los 108 blister por minuto. Adicionalmente, la balanza dinámica tiene la capacidad de sacar del proceso la plegadiza que tenga el peso diferente al estándar de la caja. A continuación, mostramos como quedaría la línea de empaque con las mejoras propuestas.

EMPAQUE CON MEJORAS

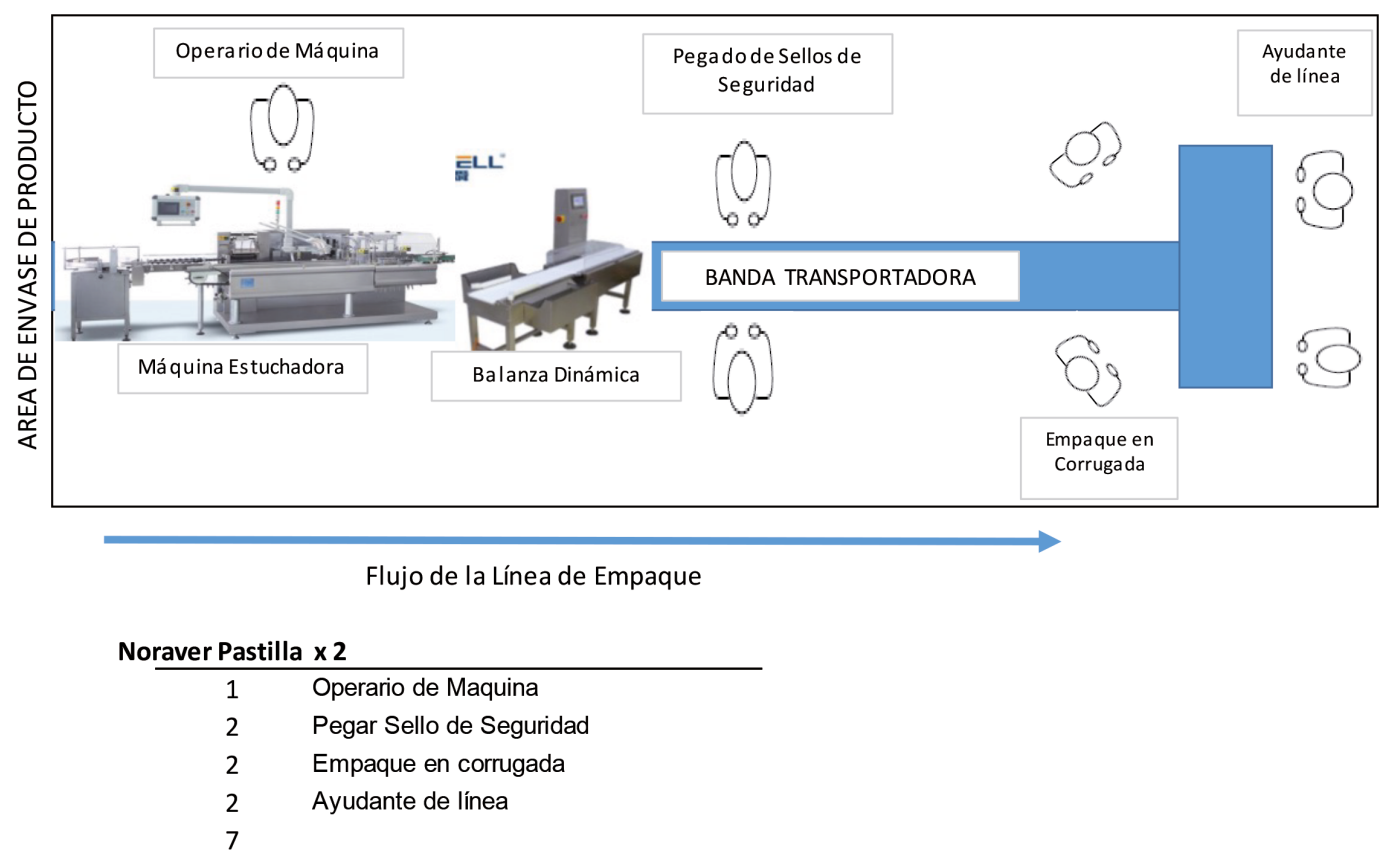

Figura 5. Propuesta de mejoramiento 1 
La línea de empaque queda distribuida de la siguiente manera (véase en la Figura 5):

La máquina estuchadora solo requiere un operario que la maneje y surta el blíster, este mismo es el encargado de verificar la configuración de la balanza dinámica; luego de este proceso, están dos operarios para pegar sello de seguridad, seguido de dos personas para empacar en corrugada y finalmente dos auxiliares de línea para estibar las cajas.

Con esta propuesta se eliminarían los $120 \mathrm{~min}$ de paros por saturación en la línea, quedando así un tiempo neto de 8,25 horas para el proceso de empaque (véase Tabla 6). De acuerdo con lo anterior, la máquina nos permite empacar la totalidad del lote en 4,5 turnos ya que empaca la todo del producto entregado por la máquina envasadora, y tenemos una disminución en la cantidad de operarios requeridos para realizar esta labor con un máximo de 7 personas (velocidad máquina envasadora 36 $\mathrm{gol} / \mathrm{min}$ para un total de 108 unidades).

216 tabletas/minuto por 60 minutos $=12.960$ tabletas por hora.

12.960 tabletas/hora por 8 horas $=103.680$ tabletas por turno.

Tabla 6. Distribución de tiempos con mejoras

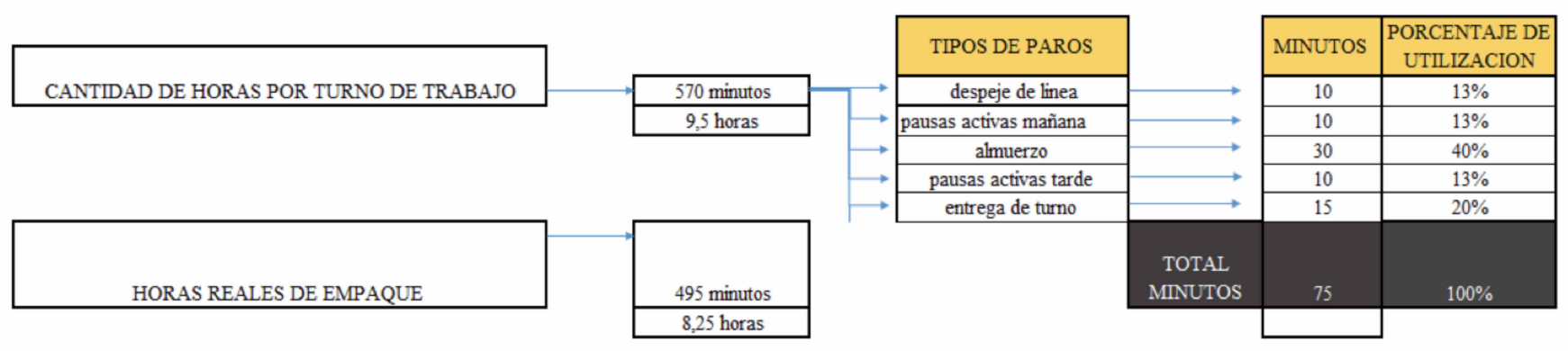

\section{Propuesta de mejoramiento 2}

Pensando en la posibilidad que no se adquiera la máquina estuchadora, se propone una alternativa para la optimización de la línea de empaque. Esta alternativa contempla la modificación del diseño de la plegadiza a semiautomática y la compra de la balanza dinámica para adecuar a la banda transportadora, así se disminuyen dos personas que pueden pasar para las líneas de efervescentes (en esta área siempre requieren personal).

En el caso de adquirir la balanza la modificación de la línea de empaque quedaría de la siguiente manera: 


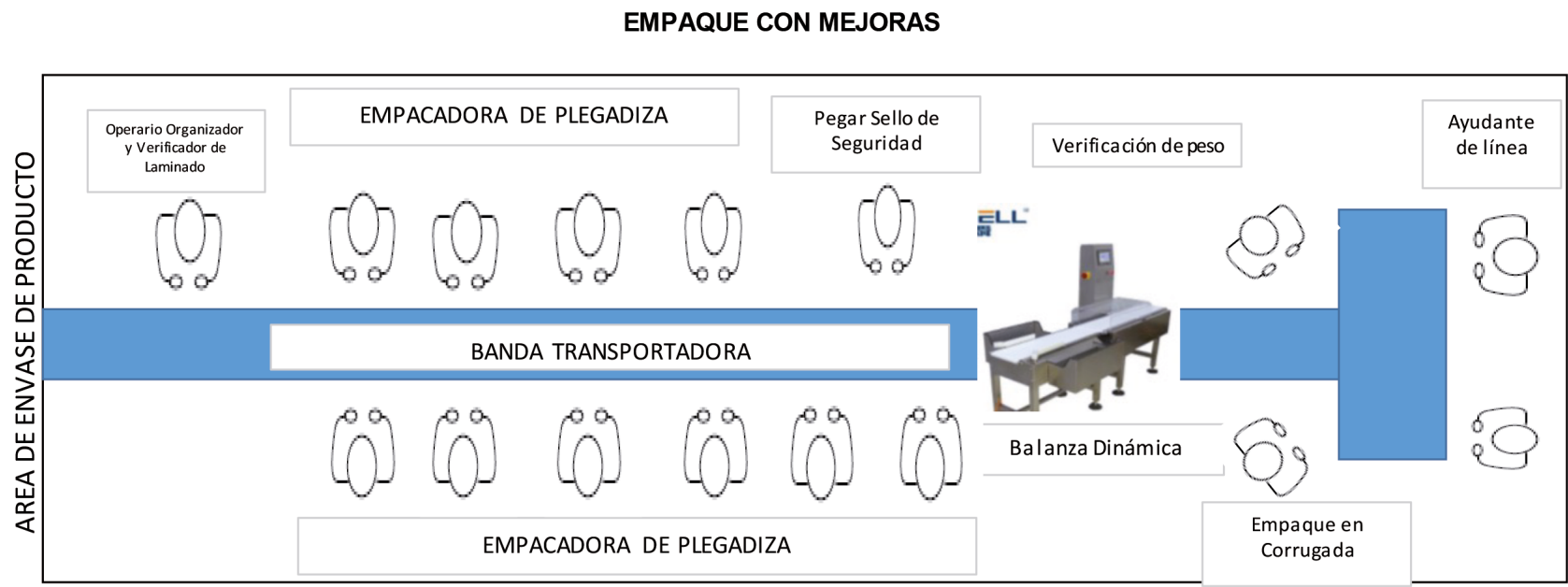

Flujo de la Línea de Empaque

Noraver Pastilla $\times 2$

$\begin{array}{ll}1 & \text { Operario Organizador } \\ 9 & \text { Empaque en plegadiza } \\ 2 & \text { Pegar Sellos de Segurida } \\ 2 & \text { Empaque en corrugada } \\ 2 & \text { Ayudante de línea } \\ & 16\end{array}$

Figura 6. Propuesta de mejoramiento 2

En esta propuesta solo saldrían dos personas; uno de los operarios de empaque en corrugada sería el encargado de configurar la balanza.

\section{FASE 4: REALIZAR LA RELACIÓN DE COSTO VS BENEFICIO DEL PROYECTO}

Para esta fase se debe tener en cuenta todos aquellos factores que generen valor en el proceso, es decir, que permitan una disminución en costo y generen un beneficio a la compañía.

\section{Recurso humano}

Para realizar la relación de costo beneficio de la propuesta de mejora del método aplicado en la actividad pegado de sello de seguridad, se tomaron los tiempos por los dos sellos de una caja determinando así que, con el nuevo método, al ejecutar 3 cajas a la vez, se logra sellar 20 unidades más que con el método actual.

Se realiza el cálculo de los costos por operario teniendo en cuenta el salario más prestaciones sociales (véase Tabla 7), a partir de este se halló el costo de 1 minuto y 1 segundo (teniendo en cuenta que los tiempos están en segundos). Una vez hallado el costo de mano de obra de este proceso se procedió a multiplicar por el tiempo de un sello por el costo de $\mathrm{MO}$ en segundo, así mismo con el tiempo por 2 sellos; adicionalmente se multiplicó el costo de $\mathrm{MO}$ de minuto por las unidades por minuto con los sellos pegados. De acuerdo con lo anterior, en la Tabla 22 se puede evidenciar que el costo por caja del método actual vs método propuesto tiene una diferencia de $-\$ 1.48$ pesos de ahorro por operación. En cuanto unidades, el costo en ahorro es de $\$ 2.846$ por las unidades por min. 
Tabla 7. Diferencia en Costos Sellos de Seguridad

\begin{tabular}{|c|c|c|c|c|c|}
\hline Costo Operario & $\$$ & $2,000,000$ & & & \\
\hline Costo Hora & $\$$ & 8,333 & & & \\
\hline Costo Min & $\$$ & 139 & & & \\
\hline Costo Segundo & $\$$ & 2.31 & & & \\
\hline Costo Pegar Sello & & Costo & $\begin{array}{c}\text { Costo por } \\
\text { Caja }\end{array}$ & & $\begin{array}{c}\text { Costo por } \\
\text { Unidades por } \\
\text { Min } \\
\end{array}$ \\
\hline 1 Caja & $\$$ & 7 & $\$$ & & $\$ \quad 555.56$ \\
\hline 3 Cajas & $\$$ & 17 & $\$$ & & 550.93 \\
\hline Diferencia & & & & & 4.63 \\
\hline
\end{tabular}

\section{Recurso tecnológico \\ Propuesta 1}

El costo beneficio de la propuesta 1 es la siguiente:

Incremento de unidades empacadas por turno: El incremento en las unidades empacadas es notablemente visible, ya que se observa una disminución bastante alta en los tiempos de empaque que permiten tener una mayor efectividad del proceso. Unidades empacadas con plegadiza normal 81000 por turno, la totalidad del lote se empaca en 6.5 turnos. Unidades empacadas con plegadiza semiautomática 98010 por turno, un incremento del $21 \%$ en la producción, esto nos permite empacar la totalidad del lote en 5 turnos de producción.

- Disminución del personal de la línea: se debe calcular primero el costo de mano de obra para ver si es viable la adquisición de la máquina estuchadora y cuál sería el tiempo de retorno de la inversión si se realiza.

En la Tabla 8 se puede ver el costo de MO, el cual es calculado con la información suministrada por recursos humanos, donde el costo de un operario de empaque es de $\$ 2.000 .000$ de salario más prestaciones sociales y extralegal. También se sacaron los costos de las máquinas propuestas en la fase anterior, el costo de adquisición es de $\$$ 100.450.000 pesos, ya que se reubican 11 operarios obteniendo así un ahorro mensual de \$22.000.000 para un retorno total de la inversión en un plazo de 4.5 meses.
Tabla 8. Costos vs Beneficio de mano de obra en la Propuesta 1

\begin{tabular}{|l|c|}
\hline Item & Costo Mes \\
\hline 7 Personas & $14,000,000$ \\
\hline
\end{tabular}

\begin{tabular}{|c|c|}
\hline Ahorro por mes & Costo Mes \\
\hline 11 Personas & $22,000,000$ \\
\hline Item & Valor Come rcial \\
\hline Máquina Estuchadora & $85,750,000$ \\
\hline Balanza Dinámica & $14,700,000$ \\
\hline Mantenimiento & $1,715,000$ \\
\hline Total Maquinaria & $102,165,000$ \\
\hline
\end{tabular}

\begin{tabular}{|l|l|l|}
\hline Retorno a la Inversión & 5.0 & Meses \\
\hline
\end{tabular}

\section{Propuesta 2}

Teniendo en cuenta que, en la fase anterior, en esta propuesta se sugirió la modificación del diseño de la plegadiza y la adaptación de la balanza dinámica a la banda transportadora para garantizar la precisión del peso del producto. En la Tabla 9 se puede observar el costo de MO, calculado conla información suministrada por recursos humanos, donde el costo de un operario de empaque es de $\$ 2.000 .000$ de salario más prestaciones sociales y extralegal. Asimismo, se calcularon los costos de la balanza dinámica propuesta en la fase anterior, el costo de adquisición es de $\$ 14.700 .000$ pesos, dando un retorno a la inversión en 4 meses.

Tabla 9. Costos vs Beneficio de mano de obra en la Propuesta 2

\begin{tabular}{|c|c|}
\hline Item & Costo Mes \\
\hline 16 Personas & $32,000,000$ \\
\hline
\end{tabular}

\begin{tabular}{|l|c|}
\hline Ahorro por mes & Costo Mes \\
\hline 2 Personas & $4,000,000$ \\
\hline
\end{tabular}

\begin{tabular}{|c|r|}
\hline Item & Valor Comercial \\
\hline Balanza Dinámica & $14,700,000$ \\
\hline
\end{tabular}

\begin{tabular}{|l|l|l|}
\hline Retorno a la Inversión & 4.00 & Meses \\
\hline
\end{tabular}




\section{CONCLUSIONES}

- Con la identificación de los problemas del proceso se lograron proponer soluciones para optimizar las actividades que presentan mayor coeficiente de variación, a saber: armado de plegadiza con $20 \%$, verificación del laminado $23 \%$, insertar tabletas en plegadiza $11 \%$, pegar sellos de seguridad $22 \%$, verificación de peso $25 \%$.

- El lead time del proceso se encuentra en 2,62 min desde el inicio del proceso hasta estibar la corrugada; la actividad con mayor porcentaje es el armado de plegadiza con el $15 \%$.

- Con la identificación de los desperdicios del proceso (mudas) se evidenció que el tiempo de espera es la muda con mayores actividades involucradas con un $35 \%$, seguido de movimientos innecesarios con $32 \%$ y mala calidad con $24 \%$.

- También se identificó que el $64 \%$ de las actividades generan valor al proceso, mientras que el $36 \%$ no generan valor, pero son necesarias para el cumplimiento de los objetivos de empaque.

- Analizando solamentelas mudasque nogeneran valor al proceso pero que son necesarias, evidenciamos que el $33 \%$ tiene tiempo de espera por cuestiones de documentación, el $33 \%$ movimientos innecesarios por actividades que deben desarrollar diferentes al proceso y, finalmente, $27 \%$ de mala calidad, ya que si la documentación solicitada y verificada no concuerda con el producto a empacar puede generar un problema de calidad.

\section{REFERENCIAS BIBLIOGRÁFICAS}

Auflage. (2016). Goldratt y la Teoría de Restricciones: El Salto Cuántico en Gerencia. Hardcover: Ibidem.

Barnes, R.M. (1962). Estudio de Movimientos y Tiempos. Madrid: Aguilar.

Cruelles Ruiz, J.A. (2013). Ingeniería Industrial. Métodos de trabajo, tiempos y su aplicación a la planificación y a la mejora continua. Juárez: Alfaomega Grupo Editor SA.

Lean Manufacturing. (18 de 01 de 2020). Valué Stream Mapping: Qué es, beneficios y cómo realizarlo. Obtenido de https://leanmanufacturing10.com/ vsm-value-stream-mapping

S. C. Graves, A. H. G. Rinnooy Kan, P. H. Zipkin. "Handbooks in Operations Research and Management Science". Logistics of Production and Inventory. Elsevier Science Publishers. North Holland. The Netherlands. Vol. 4. 1993. pp. 3-50

Tecnoquímicas. (10 de 02 de 2019). Metodología de las 5s. Obtenido de https://www.tqconfiable.com/producto-homeindustrial/gestion-y-control-de-calidad/metodologia-de-las-5s/

Lagoy, A. E., \& Zúñiga, J. F. (2015). Ingeniería Industrial. Métodos y tiempos con manufactura ágil. Buenos Aires: Alfaomega Colombiana.

Serrano, A. T. (2012). Control interno y sistema de gestión de calidad. Lima: Ediciones de la U.

Socconini, L. (2019). Lean Manufacturing. Paso a Paso. Marge Books.

Hernández Matías, J.C. y Vizán Idoipe, A. (mayo 2013). Lean manufacturing, conceptos, técnicas e implantación. Medio ambiente, industria y energía. Recuperado de https://www.eoi.es/es/savia/publicaciones/20730/lean-manufacturing-concepto-tecnicas-e-implantacion 


\section{AUTORES}

\section{Diomar Aurora Dorado}

Tecnóloga en Producción Industrial UNIAJC. Aspirante al título de Ingeniero industrial de la UNIAJC. Integrante del semillero Simec. Correo: diomar928@hotmail.com

\section{Julián David Cifuentes}

Tecnólogo en Automatización Industrial del Sena. Aspirante al título de Ingeniero industrial de la UNIAJC. Integrante del semillero Simec. Correo: julian_cifuentes1@hotmail.com

\section{Alexander Lozano}

Tecnólogo en Producción Industrial UNIAJC. Aspirante al título de Ingeniero industrial de la UNIAJC. Integrante del semillero Simec. Correo: 1.r.alex.19@ gmail.com

\section{Carlos Andrés Nieto}

Ingeniero Mecánico de la Universidad Autónoma de Occidente, con estudios en automatización, gerencia financiera, MBA en administración con énfasis en gerencia estratégica de la Universidad ICESI. Profesor tiempo completo de la Facultad de Ingeniería en la Institución Universitaria Antonio José Camacho, perteneciente al grupo de investigación Inteligo. Correo: canieto@admon. uniajc.edu.co 